British National Formulary. London \& Oxford: BMA \& The Pharmaceutical Press.

Trohman, R. D (1988) Amiodarone induced delirium. Annols of Internal Medicine, 108, 68-69.

A. Ambrose, E. Sallb Hollins Park Hospital, Hollins Park, Hollins Lane, Winwick, Warrington WA2 8WA

\section{Psychological treatments for hypochondriasis}

Sir: Clark et al's (1998) loose use of terms is misleading. Their "cognitive" therapy was in fact cognitive-behavioural therapy (CBT) with (p. 219) "A mixture of cognitive and behavioural techniques" including "behavioural experiments" by imaginal exposure ("inducing symptoms by deliberate body focusing or dwelling on fearful thoughts"), live exposure ("increasing engagement in activities that were avoided because of illness beliefs (for example, exercise)"), and "response prevention for repeated bodily checking and prevention of reassurance seeking . . . others who were normally involved in the provision of repeated reassurance were included in the response prevention programme and were given instructions in how to deal correctly with any further requests for reassurance". Homework included exposure and response prevention (ERP).

In contrast," "behavioural stress management" included only weak exposure without mention of 'cognitive' therapy's strong behavioural components of: ERP in the first few sessions; behavioural experiments and response prevention by patients and others to deal with checking and reassurance seeking; and exposure homework. It did include anti-exposure reassurance ("remind patients that previous physical investigations had proved negative and their doctor was convinced they did not have a serious illness"). The procedure is best termed stress management with a small behavioural component late in therapy.

The design's having more behavioural (ERP) experiments in the cognitive $(80 \%)$ than in the behavioural therapy $(0 \%)$ sessions shows in Table 1 . The Table does not mention exposure homework, but the description (see above) suggests this too was advised more in the cognitive than the behavioural sessions. Because the authors' cognitive therapy was also more behavioural (had more ERP) than their behavioural treatment, their design cannot support the claim that cognitive therapy was a specific treatment, unlike behavioural stress management. They compared CBT (cognitive restructuring plus ERP) on the one hand with stress management including limited exposure and additional methods on the other. The early superiority of their CBT (which was not sustained) could be explained by its greater use of ERP than the stress management protocol which introduced exposure later in treatment.

It is possible that cognitive therapy alone, without behavioural experiments and ERP, may have produced similar improvement, but the study has no such contrast group. What was specific about a form of cognitive therapy that included strong behavioural methods in a design which had no treatment group that omitted both cognitive and behavioural components?

Clark et al's design is out of date, as controlled studies have found in several anxiety disorders, including hypochondriasis, that exposure alone and cognitive therapy alone were each therapeutic in their own right. In depressive disorders too, purely behavioural (without cognitive) methods were just as helpful. None of these controlled studies is cited.

Clark et al's preoccupation with cognitive effects leads them to ignore recent findings that neither cognitive nor behavioural therapy is crucial for improvement. Sufficient yes, necessary no. One or the other can do the trick, and each may be an unwitting way of using other effective ingredient(s) that are as yet unidentified. Future studies are more likely to advance knowledge if they separate cognitive from behavioural components and test whether they work by similar or different mechanisms or in ways that are neither cognitive nor behavioural.

As an aside, on Fig. 1's measure none of the follow-up differences between the two treatments was significant.

Chark, D M., Salkorakis, R. M., Hackman, A., et al (1990) Two psychological treatments for hypochondriasis. A randomised controlled trial. British journol of Psychiotry 173, 218-225.

I. Mariss Institute of Pychiatry, De Crespigny Park, London SE5 8AF

Authors' reply: Our controlled trial demonstrated that two new treatments, developed by our group, produce substantial improvements in hypochondriasis. Professor Marks quibbles with the labels chosen for the treatments and our use of the term "specific treatment effect". Personally, we are more concerned with effectiveness than with labels. However, our terminology was not inappropriate.

The term 'cognitive therapy' was introduced over 30 years ago and from the start denoted a cognitive theory-based treatment involving verbal disputation and behavioural procedures, both of which had the explicit aim of changing patients' dysfunctional beliefs (see Beck, 1970). Our cognitive therapy for hypochondriasis has these characteristics. Some people prefer the term cognitive-behavioural therapy (CBT). We chose cognitive therapy not because we think our behavioural procedures are unimportant: quite the contrary. Instead, it was because the term CBT is used in a variety of different, and potentially confusing, senses. For some people it equals cognitive therapy as defined above. For others, such as Marks, it includes a mixture of procedures that are each given with different rationales, viz. an anxiety habituation rationale for exposure and a belief change rationale for verbal disputation.

The term 'specific treatment effect' also has a long-standing meaning, which we adhered to. At least since Gelder et al's seminal paper (1973) on specific and nonspecific effects in psychotherapy, the term has been used to denote a demonstration that the effects of a therapy cannot be accounted for simply by a series of specified procedures that would be present in any well-conducted psychological treatment, irrespective of orientation. Our cognitive therapy programme clearly passed this test as it was superior to behavioural stress management on 7 out of 10 hypochondriasis measures at post-treatment, despite behavioural stress management involving the same repeated assessments, being administered by the same therapists for the same amount of time, involving systematic out-of-session homework, and being rated as equally credible by patients. This demonstration of specificity seems rather more convincing than Marks' own claims for specificity in his recent trial of treatments for post-traumatic stress disorder (PTSD) (Marks et al, 1998). In that trial, exposure was only superior to the control treatment (relaxation) on three out of nine primary PTSD measures and there was no evidence that patients 